\title{
Millimeter-wave close proximity high-speed data transfer system
}

\author{
Tadao Nakagawa ${ }^{1 \text { a) }}$, Hideki Toshinaga ${ }^{1}$, Toshimitsu Tsubaki ${ }^{1}$, \\ Tomohiro Seki ${ }^{2}$, and Masashi Shimizu ${ }^{1}$ \\ ${ }^{1}$ NTT Network Innovation Laboratories, NTT Corporation, \\ Yokosuka, Kanagawa 239-0847, Japan \\ ${ }^{2}$ College of Industrial Technology, Nihon University, \\ 1-2-1 Izumicho, Narashino, Chiba 275-8575, Japan
}

a)nakagawa.tadao@lab.ntt.co.jp

\begin{abstract}
This paper presents the system concept, transceiver architecture, and control sequence for a millimeter-wave $(60-\mathrm{GHz})$ band close proximity high-speed data transfer system. The communication range and the use case are limited to achieve fast link setup time and a stable point-to-point connection. Prototype equipment developed for the system includes three types of wireless transceivers; cooperative operation among them makes it possible to reduce the link setup time and limit the communication range. The system's control sequence enables the link setup time to be reduced from 7 seconds to 0.2 seconds.
\end{abstract}

Keywords: millimeter wave, $60 \mathrm{GHz}$, close proximity high-speed data transfer, link setup time, point-to-point connection

Classification: Wireless Communication Technologies

\section{References}

[1] E. Perahia, C. Cordeiro, M. Park, and L. L. Yang, "IEEE 802.11ad: defining the next generation multi-Gbps Wi-Fi," Proc. IEEE 2010 Consumer Communications and Networking Conference (CCNC 2010), pp. 1-5, 2010. DOI:10.1109/ CCNC.2010.5421713

[2] T. Baykas, C.-S. Sum, Z. Lan, J. Wang, M. A. Rahman, H. Harada, and S. Kato, "IEEE 802.15.3c: the first IEEE wireless standard for data rates over $1 \mathrm{~Gb} / \mathrm{s}$," IEEE Commun. Mag., vol. 49, pp. 114-121, Jul. 2011. DOI:10.1109/MCOM. 2011.5936164

\section{Introduction}

Mobile traffic has increased significantly in recent years with the widespread use of smartphones and tablets and their increasingly high volume content. It is expected that wireless LANs will enable traffic offloading, but in urban areas their effective throughput is rapidly degraded as the user number increases. Millimeter-wave (60-GHz) band wireless systems have a wide bandwidth of about $9 \mathrm{GHz}$ and great 
potential in terms of capacity $[1,2]$. There are, however, some problems in using the millimeter-wave band, including link setup time and communication topology. We propose a millimeter-wave close proximity high-speed data transfer system to solve these problems and to provide a comfortable data transmission service. In this paper we describe the system concept, transceiver architecture, and the performance of prototype equipment developed for the system.

\section{System concept}

The use case of our target system is high-speed data download/upload services that are carried out at kiosk terminals, which are located in many types of public spaces including convenience stores, train stations, and airports. Our target system has the following features:

1) Link setup time is less than 1 second:

Link setup includes association and authentication between a kiosk terminal and a mobile terminal. When the transmission rate of wireless links becomes high, the link setup time will remain noticeably high. For example, a video file with a viewing time of 1 hour that is compressed with a H.265 video compression standard has a data size of about $400 \mathrm{MB}$. This results in file download/upload time of about 3.2 seconds at a transmission rate of $1 \mathrm{Gbit} / \mathrm{s}$, while the link setup time of the existing millimeter-wave systems is generally more than 5 seconds. Thus the link setup time should be reduced to provide a short file transfer period. 2) The communication range is less than $10 \mathrm{~cm}$ :

This short-range system ensures that the system's communication topology is a point-to-point connection, whereas the existing millimeter-wave systems including IEEE 802.11ad and IEEE 802.15.3c may have point-to-multipoint connections since their communication ranges are several meters [1, 2]. A point-to-point connection has a larger effective throughput than point-to-multipoint connections. The effective throughput of user terminals at an access point or a base station lessens as the number of users per cell increases.

In order to achieve these features, the system we propose combines multiple wireless systems. We hereafter refer to it as the "proposed multiple wireless system" and describe it in the following section.

\section{Configuration}

The transmitter configuration for our proposed multiple wireless system is shown in Fig. 1. The kiosk terminals and mobile terminals have high-speed caches between which data transfer is carried out. Both the kiosk terminals and mobile terminals have three kinds of wireless transceivers: millimeter-wave, Wi-Fi wireless LAN, and NFC (near field communications).

The transmitter control sequence is shown in Fig. 2. When a user with a mobile terminal enters a Wi-Fi communication zone (about $10 \mathrm{~m}$ ), Wi-Fi connection and authentication are carried out. The authentication information is used in millimeterwave connections and NFC connections. When a mobile terminal enters an NFC communication zone (about $10 \mathrm{~cm}$ ), the terminal is identified. Millimeter-wave data transfer is carried out for the identified mobile terminals. 


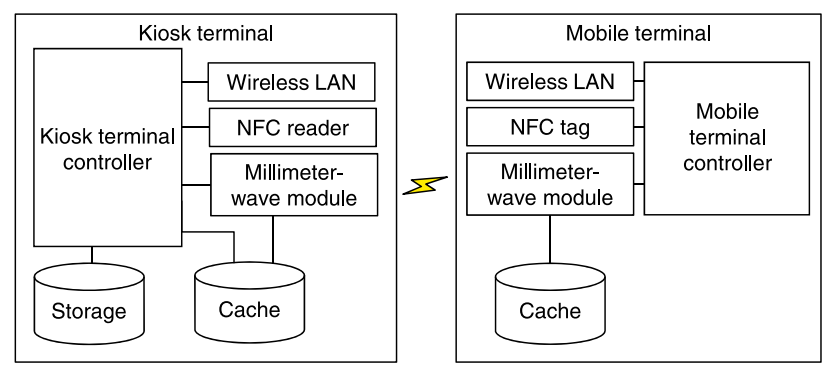

Fig. 1. Transmitter configuration for the proposed multiple wireless system.

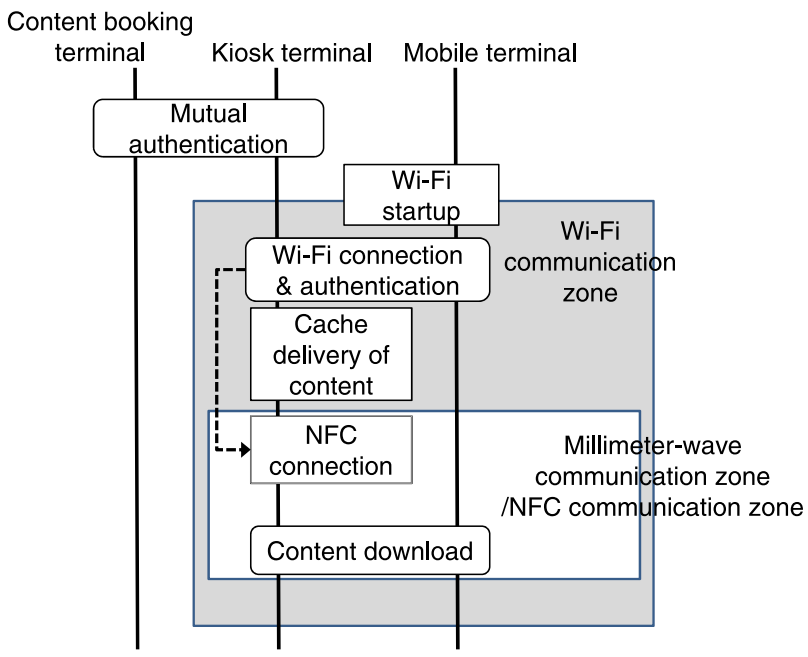

Fig. 2. Transmitter control sequence.

In this sequence the link setup, including association and authentication between a kiosk terminal and a mobile terminal, will have been finished before a mobile terminal approaching the contact area of a kiosk terminal starts acting. NFC is used to limit the communication range of millimeter-wave data transfer. This is because the NFC antennas operate through electromagnetic induction, which makes it easy to limit the communication range to less than that for antennas that operate through radio wave emission. If the equivalent isotropically radiated power (EIRP) of a millimeter-wave module is set so that the communication range is $10 \mathrm{~cm}$, the millimeter-wave connection within $10 \mathrm{~cm}$ may be unstable due to a tilt of a mobile terminal or a position alignment error between a mobile terminal and a kiosk terminal. However, this problem does not occur when the communication range is limited by using NFC.

\section{Prototype equipment}

We have developed prototype equipment including kiosk terminals and mobile terminals that have millimeter-wave $(60 \mathrm{GHz})$ modules in them as shown in Fig. 3. Between the millimeter-wave modules the transmission rate was $2.5 \mathrm{Gbit} / \mathrm{s}$ and between the caches it was $1 \mathrm{Gbit} / \mathrm{s}$, including the read and write times of the caches. 


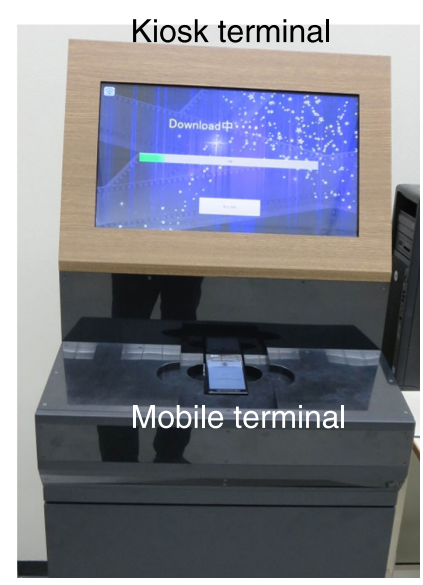

Fig. 3. Prototype equipment for the proposed multiple wireless system.

Measured performance comparison between a conventional millimeter-wave module (i.e., one for millimeter waves only) and the proposed multiple wireless system is as follows:

1) Conventional millimeter-wave module:

The link setup time is 7 seconds. The communication range is $50 \mathrm{~cm}$.

2) Proposed multiple wireless system:

The link setup time is 0.2 seconds. The communication range is $10 \mathrm{~cm}$.

As the comparison shows, the latter has much faster link setup time at the designed lower communication range.

\section{Conclusion}

In this paper, we described the system concept, transceiver architecture, and control sequence for a millimeter-wave $(60-\mathrm{GHz})$ band close proximity high-speed data transfer system. The communication range and the use case are limited to achieve fast link setup time and a stable point-to-point connection. Prototype equipment developed for the system includes three kinds of wireless transceivers, and cooperative operation among them makes it possible to reduce the link setup time and limit the communication range. The system's control sequence provided $1 \mathrm{Gbit} / \mathrm{s}$ transmission rate and enabled the link setup time to be reduced from 7 seconds to 0.2 seconds with the targeted communication range of $10 \mathrm{~cm}$. 